\title{
Differential birefringence in Bragg gratings in multicore fiber under transverse stress
}

\author{
Manuel Silva-Lopez, Cheng Li, William N. MacPherson, Andrew J. Moore, \\ James S. Barton, and Julian D. C. Jones \\ Applied Optics and Photonics Group, School of Engineering and Physical Sciences, Heriot-Watt University, Edinburgh EH14 4AS, UK
}

Donghui Zhao, Lin Zhang, and Ian Bennion

Photonics Research Group, Electronic Engineering and Computer Science Division, Aston University, Birmingham B4 7ET, UK

Received May 20, 2004

\begin{abstract}
We present experimental measurements of the peak splitting of the reflection spectra of fiber Bragg gratings as a result of birefringence induced by transverse loading of a multicore fiber. Measurements show that the splitting is a function of the applied load and the direction of the load relative to the azimuth of the fiber. A model for calculating the stress in the fiber that is due to an applied load is in good agreement with our experimental observations. (C) 2004 Optical Society of America

OCIS codes: $060.2400,060.2370$.
\end{abstract}

An important application of optical fiber Bragg gratings (FBGs) is in the measurement of longitudinal strain, in the direction of the axis of the fiber. However, there is interest in also being able to measure components of the strain transverse to the fiber, ${ }^{1}$ e.g., in the diagnosis of the strain field within composite materials. Transverse strain modifies the refractive-index profile of the fiber, thus providing a transduction mechanism. The stress field in transversely loaded fiber was originally investigated to estimate possible mode coupling effects arising from transverse stress perturbations of the refractive-index profile. ${ }^{2}$ This was followed by studies of the birefringence induced by transverse stresses that were due to external $\operatorname{load}^{3}$ or internal thermally induced stresses in highly birefringent (hi-bi) fiber. ${ }^{4,5}$ The response of FBGs in hi-bi fiber to transverse loading was reported by Lawrence et al., ${ }^{1}$ who showed that the Bragg wavelength shifts corresponding to the two orthogonal polarization modes varied approximately linearly with transverse load. In general, the birefringence increased with load, but a decrease occurring for load orientations near parallel to the slow axis has been observed. Ye et al. used a polarization-maintaining fiber coupler ${ }^{6}$ to detect the two polarization modes separately, avoiding the problem of resolving the two Bragg peaks when their separation decreases with load. Abe et al. recently reported ${ }^{7}$ the use of two superimposed FBGs in hi-bi fiber to allow simultaneous measurement of transverse strain and temperature.

In this Letter we describe experimental measurement of birefringence in FBGs written in a multicore fiber (MCF) subject to transverse loading. Our objective was to demonstrate measurement not only of magnitude of the transverse strain but also its direction. In contrast with conventional hi-bi fiber, MCF has the advantage that the cores are not limited to a central location and FBGs in MCF cores can sample the transverse stress field at several points in the fiber cross section. When the fiber is subject to transverse load, the transverse stress is greatest close to the edge of the cladding along the diameter aligned with the load axis and is least for cores farthest from the load axis. Hence, by observing the differential birefringence between cores revealed by the grating spectra, we can infer the load magnitude and orientation.

The MCF was fabricated from a preform into which four holes were drilled to accept doped glass rods that formed the cores of the fiber. The boron codopant was chosen to enhance the photosensitivity of this fiber specifically for the purpose of writing gratings. The diameter of the preform and location of the holes were such that, after being pulled, the resulting fiber was $125 \mu \mathrm{m}$ in outer diameter with a square matrix of cores with a core spacing of $50 \mu \mathrm{m}$. Each core was single mode at $1550 \mathrm{~nm}$ and was sufficiently distant from the outer cores that core-to-core coupling was not evident.

The intrinsic photosensitivity of this MCF permits UV inscription of the FBG structures in the cores without hydrogenation of the fiber, a common treatment for grating fabrication in standard fibers and in the noncircular four-core fibers that we have used in previous work. ${ }^{8}$ We have produced high-efficiency FBGs in the present $\mathrm{MCF}$, using a standard phase mask scanning technique ${ }^{9}$ and a frequency-doubled Ar laser with a $95-\mathrm{mW}$ output at $244 \mathrm{~nm}$. In the fabrication the UV beam was scanned at a speed of $0.07 \mathrm{~mm} \mathrm{~s}^{-1}$ over a $10-\mathrm{mm}$ length of the fiber. To write gratings selectively in the respective cores we mounted the fiber on a rotation stage to accommodate the setting of the fiber azimuth. Both single- and double-exposure methods were employed to write grating structures in one or more cores. The latter involves a second UV exposure after rotating the fiber by $180^{\circ}$ following the first exposure.

The MCF containing FBGs was interrogated with the configuration shown in Fig. 1. The MCF containing the FBGs to be tested was fusion spliced onto a length of MCF that forms part of a fan-out ${ }^{8}$ assembly that interfaces four standard SMF-28 input fibers to 


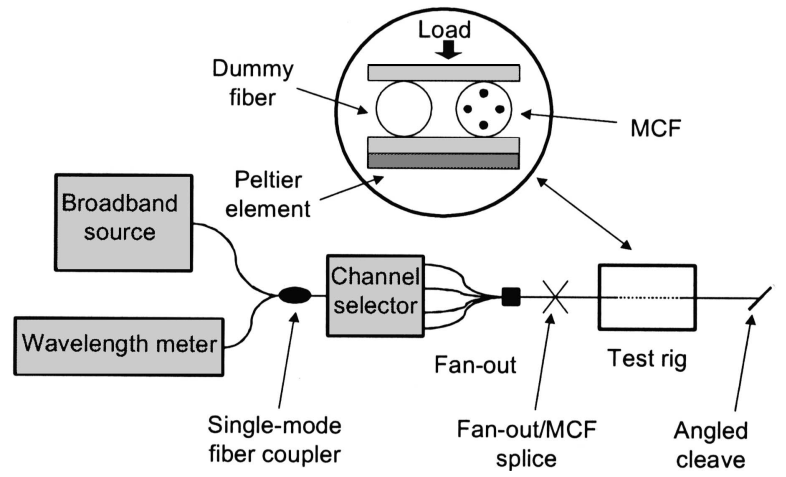

Fig. 1. Interrogation scheme used to measure MCF FBG reflection spectra. The MCF is located in a temperaturestabilized load test rig capable of applying loads up to $250 \mathrm{~N}$ to the $50-\mathrm{mm}$ length of MCF fiber.

one MCF output, such that each input addresses only one of the MCF cores. The MCF to MCF fusion splicing procedure was similar to that used for conventional hi-bi fiber (e.g., bow-tie fiber) such that in addition to lateral and longitudinal alignment of the fibers the rotation must be controlled to ensure that the cores of each fiber are correctly aligned. A fusion splicer capable of imaging the ends of the fiber to facilitate the rotational alignment was used, thus achieving splice losses typically better than $1.0 \mathrm{~dB}$. The four input fibers could be selected individually by use of a fiber switch channel selector, thereby enabling the reflection spectrum from each of the four cores to be interrogated sequentially by use of a broadband, nonpolarized source (wavelength range 1530-1560 nm and an output power of $\cong 11 \mathrm{dBm}$ ) and a wavelength meter with 14-pm resolution.

The fiber was positioned between two flat polished aluminum plates so that it was parallel to a second dummy fiber. This configuration ensured that the applied load was distributed uniformly by constraining the two plates to remain parallel under loading. Transverse stress was applied by adding masses to the top aluminum plate via a system of loading plates and linear bearings to ensure that the load was evenly distributed. The length of sensing fiber under load was $50 \mathrm{~mm}$, and it could be rotated about its central axis to allow the direction of the applied transverse load to be varied relative to the fiber axis. The temperature was controlled with Peltier elements and closed-loop control units, and the fiber was coated with silicone oil for good thermal contact with the temperature-stabilized plates. Finally, we applied a small axial tension to maintain a straight fiber in the test section.

Before completing the fiber installation it was necessary to verify the MCF orientation. By illuminating each core with a visible red laser and using a microscope to observe the light scattered from the core, it was possible to deduce the azimuth of the fiber. By rotating the fiber, we observed the relative position of each of the four cores and by focusing the microscope it was possible to infer the core height, thus unambiguously revealing the fiber orientation.

The reflected spectra of the FBG under an applied load of $205 \mathrm{~N}$ can be seen in Fig. 2. It is evident that, as the transverse load is increased, the reflected spectrum splits to form two peaks corresponding to the strain-induced birefringence, each representing one of the polarization eigenstates and whose separation is proportional to load. The two peaks are of equal magnitude, but for reasons that we have not explained they are of unequal width. From mechanical considerations, cores lying close to the load axis ( $y$ axis) experience greater strain than the orthogonal ones, consistent with the observed spectra. The FBG spectra in cores 3 and 4 were equivalent to the spectra in cores 1 and 2, respectively, indicative of the axial symmetry of the stress field over the cross section of the fiber. The structure on the grating reflection spectrum is attributed to the grating writing process, which was not sufficiently controlled to yield an ideal Gaussian reflection profile.

Incremental loading applied to the fiber induces increasing birefringence in the cores, resulting in the peak splitting shown in Fig. 3 . The grating wavelength was defined as the wavelength of maximum reflected power. Although there are more robust ways of defining the grating wavelength, such as peak fitting or centroid calculation, the maximum power
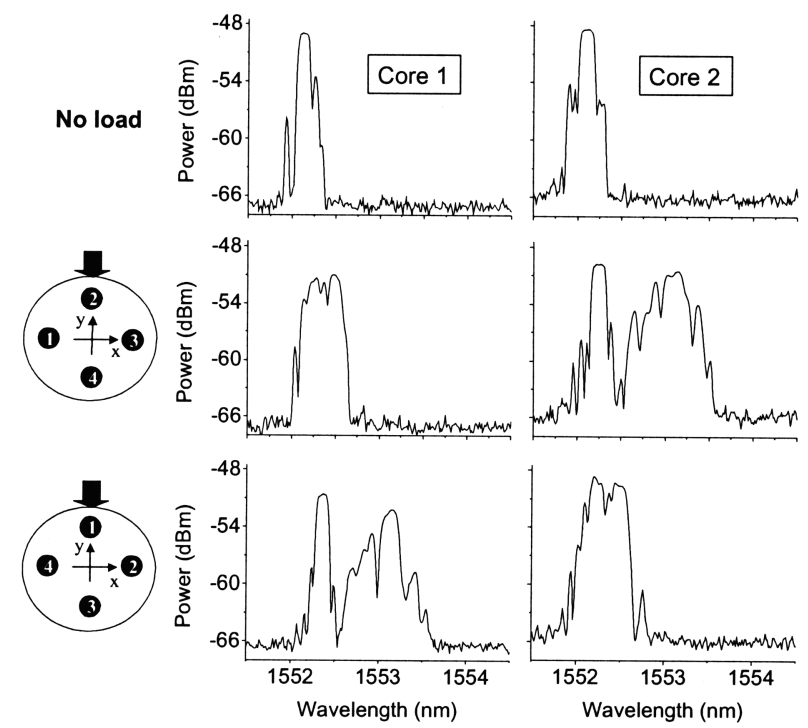

Fig. 2. Reflected FBG spectra for different orientations of MCF under a load of $205 \mathrm{~N}$.

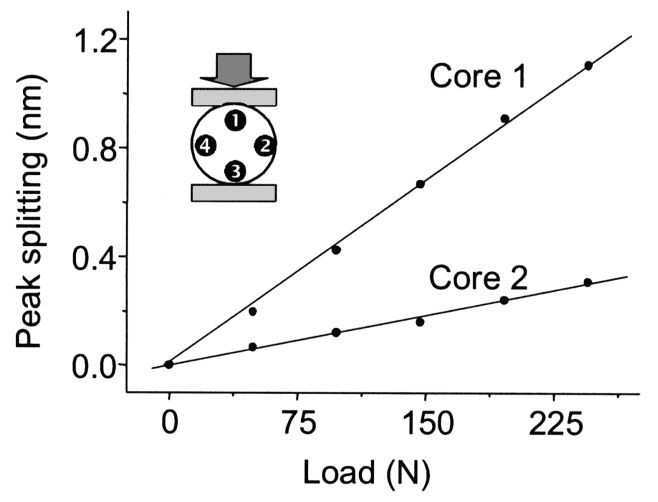

Fig. 3. Birefringence-induced peak splitting due to increasing load. 


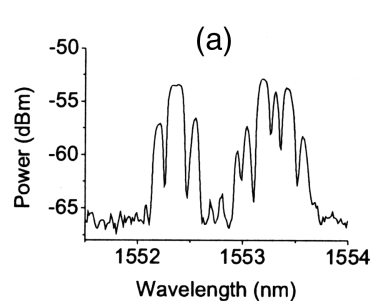

(b)
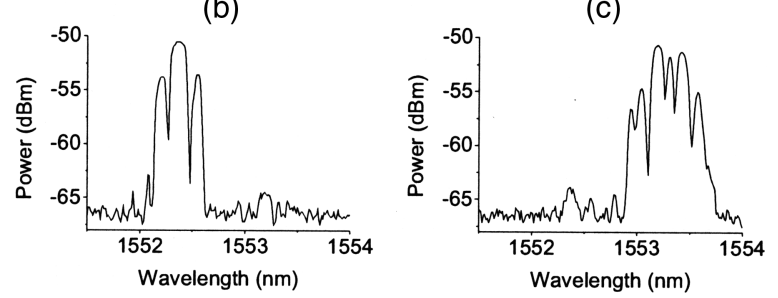

Fig. 4. Typical results illustrating the combined measurement of (a) reflection peaks and (b), (c) the effect of separating the polarization states by use of a polarization controller and a beam splitter.

technique was deemed sufficient for this measurement. From Fig. 3, the ratio of the peak splitting due to the stress difference between cores 1 and 2 (with the orientation shown) is 3.65 .

To confirm that the peak splitting was indeed due to induced birefringence we modified the experimental configuration shown in Fig. 1 to include a polarization controller after the broadband source, and a polarization splitter before the wavemeter to measure the reflection spectra in orthogonal polarization axes. The polarization splitter allowed the two eigenstates to be monitored independently, as shown in Fig. 4. Figure 4(a) shows the reflection spectrum without polarization splitting, and Figs. 4(b) and 4(c) show the spectra obtained from the two orthogonal polarization states. This clearly illustrates that the peak splitting is due to the birefringence induced in the fiber cores.

To understand better the behavior of the MCF gratings under transverse loading we investigated it theoretically by use of the model developed by Gafsi and El-Sherif. ${ }^{10}$ The calculated results show that the expected birefringence ratio between the top gratings (2 and 4 ) and the side gratings ( 1 and 3 ) to be 3.77 for a fiber oriented as illustrated in Fig. 3. This is in good agreement with the experimental result of 3.65 obtained from data shown in Fig. 3. The model predicts that when the fiber is turned incrementally through $180^{\circ}$, the peak splitting ratio between the two adjacent gratings changes continuously from the maximum of 3.77 to a minimum of 0.27 (at $90^{\circ}$ from the starting position), before returning to the maximum value. This implies that the differential birefringence between cores can be used to measure the transverse loading applied in an arbitrary direction. An advantage of the proposed technique is that it is based on the splitting of the reflection peak rather than its absolute value and is hence relatively insensitive to both temperature and axial strain.

From the model we also observed that the birefringence ratio strongly depends on the core-core spacing. The ratio of 3.77 for the current $50-\mu \mathrm{m}$ core-core separation is expected to rise to 21.0 for a core-core separation of $72 \mu \mathrm{m}$, while maintaining the $125-\mu \mathrm{m}$ outside diameter. In this case the top grating will be closer to the fiber surface and will experience a larger transverse stress, increasing the sensitivity to loading and orientation.

In summary, in this experimental study, which we believe provides the first report of transverse loading of FBGs in MCF, we have observed peak splitting in the FBG reflection spectrum as a function of applied transverse load and orientation. Further experiments proved that this splitting is due to stressinduced birefringence, and the results agree well with a model of the stress in the fiber. This experiment demonstrates the potential of MCF as a multiaxis load sensor.

The authors acknowledge the UK Engineering and Physical Science Research Council (EPSRC) and UK Defence Science and Technology Laboratory for provision of funding through the Joint Grant Scheme, and W. N. MacPherson and A. J. Moore thank the EPSRC for funding via the Advanced Fellowship Programme. The authors also thank G. Fleming (NASA Langley) for supplying the MCF. W. N. MacPherson's e-mail address is w.n.macpherson@hw.ac.uk.

\section{References}

1. C. M. Lawrence, D. V. Nelson, E. Udd, and T. Bennett, Exp. Mech. 39, 202 (1999).

2. P. D. Gianino and B. Bendow, Appl. Opt. 20, 430 (1981).

3. K. Okamoto, T. Hosaka, and T. Edahiro, IEEE J. Quantum Electron. QE-17, 2123 (1981).

4. M. P. Varnham, D. N. Payne, A. J. Barlow, and R. D. Birch, J. Lightwave Technol. LT-1, 332 (1983).

5. P. L. Chu and R. A. Sammut, J. Lightwave Technol. LT-2, 650 (1984).

6. C. C. Ye, S. E. Staines, S. W. James, and R. P. Tatam, Meas. Sci. Technol. 13, 1446 (2002).

7. I. Abe, O. Frazao, R. N. Nogueira, H. J. Kalinowski, J. L. Pinto, and J. L. Santos, in Proceedings of the 16th International Conference on Optical Fiber Sensors (Institute of Electronics, Information and Communication Engineers, Tokyo, 2003), pp. 96-99.

8. G. M. H. Flockhart, W. N. MacPherson, J. S. Barton, J. D. C. Jones, L. Zhang, and I. Bennion, Opt. Lett. 28, 387 (2003).

9. K. O. Hill, B. Malo, F. Bilodeau, D. C. Johnson, and J. Albert, Appl. Phys. Lett. 62, 1035 (1993).

10. R. Gafsi and M. A. El-Sherif, Opt. Fiber Technol. Mater. Devices Syst. 6, 299 (2000). 